\title{
Scalar Electrodynamics and Primordial Magnetic Fields
}

\author{
Francisco D. Mazzitelli \\ Departamento de Física, Facultad de Ciencias Exactas y Naturales \\ Universidad de Buenos Aires- Ciudad Universitaria, Pabellón I \\ 1428 Buenos Aires, Argentina \\ and \\ Instituto de Astronomía y Física del Espacio \\ Casilla de Correo 67-Sucursal 28 \\ 1428 Buenos Aires, Argentina \\ Federico M. Spedalieri \\ Departamento de Física, Facultad de Ciencias Exactas y Naturales \\ Universidad de Buenos Aires- Ciudad Universitaria, Pabellón I \\ 1428 Buenos Aires, Argentina
}

\begin{abstract}
A primordial magnetic field may be generated during an inflationary period if conformal invariance is broken. We reexamine and generalize previous results about the magnetic field produced by couplings of the form $R^{n} F_{\mu \nu} F^{\mu \nu}$. We show that the amplitude of the magnetic field depends strongly on $n$. For adequate values of $n$ the field produced can serve as seed for galactic magnetic fields. We also compute the effective interaction between the electromagnetic field and the geometry in the context of scalar QED (with and without classical conformal invariance). In both cases, the amplitude of the magnetic field is too small to be of astrophysical interest.
\end{abstract}




\section{INTRODUCTION}

Magnetic fields play an important role in a variety of astrophysical situations. There is enough evidence for the existence of intragalactic magnetic fields [1, 2], with an amplitude of $10^{-6} G$ and uniform on a scale of $10 \mathrm{kpc}$. It is not completely clear how these magnetic fields

were generated. A plausible explanation is that some kind of dynamo effect [3] could have amplified a pre-existent magnetic field. But then the question is about the mechanism that produced this 'seed' field.

An attractive suggestion is that it has a primordial origin and could have been produced in the early universe during an inflationary period [4 6]. Denoting by $r$ the energy density of the magnetic field relative to the energy density of the cosmic microwave background radiation, $r=\frac{\rho_{B}}{\rho_{\gamma}}$, a pregalactic magnetic field caracterized by $r \simeq 10^{-34}$ is needed in order to explain the present value of $r \simeq 1$.

As pointed out by Turner and Widrow [4], it is not possible to produce the required seed field with the usual Maxwell Lagrangian. The reason is conformal invariance. Indeed, in a conformally invariant theory $B$ decreases as $\frac{1}{a^{2}}$, where $a$ is the scale factor of the Robertson-Walker metric. During (exponential) inflation, the total energy density in the universe is constant, and the magnetic field energy density is strongly suppressed, giving $r(\lambda)=10^{-104}\left(\frac{1 M p c}{\lambda}\right)^{4}$.

Conformal invariance can be broken in different ways. From a phenomenological point of view, one can consider [4] couplings of the form $R A_{\mu} A^{\mu}$. This type of interaction-terms give rise to the required seed, but, not being gauge-invariant, are theoretically unappealing. In string-inspired models, conformal invariance is broken by the coupling between the electromagnetic field and the dilaton. This coupling may produce the seed field [5]. Finally, one can consider gauge-invariant couplings of the form $\frac{e^{2}}{m^{2}} R F_{\mu \nu} F^{\mu \nu}$. However, the seed field produced is extremely small [4].

In this paper we will reexamine the generation of primordial magnetic field due to the above mentioned gauge-invariant couplings. These terms appear due to quantum effects 
when taking into account one loop corrections for QED in curved spaces [7] (throughout the paper we will work in the context of scalar QED). In an expansion in powers of $\frac{\mathcal{R}}{m^{2}}$ (Schwinger DeWitt Expansion, SDWE [8]) U, one expects the effective action to contain couplings the form $\frac{\mathcal{R}^{n}}{m^{2 n}} F_{\mu \nu} F^{\mu \nu}$. But as already mentioned in Ref. [⿴囗才], during the inflationary period one tipically has $\mathcal{R} \gg m^{2}$. Therefore, there is no reason to keep the lowest order contribution $n=1$, and it is of interest to investigate more general couplings.

In the next Section we will compute the primordial magnetic field produced by couplings of the form $\frac{R^{n}}{m^{2 n}} F_{\mu \nu} F^{\mu \nu}$. For $n=1$, our results are considerably smaller than those of Ref. [4. The discrepancy is due to an overestimation of the value of $r$ at first horizon crossing. However, we will show that the amplitude of the magnetic field depends dramatically on $n$. In particular, for adequate values of $n$, it is possible to generate a sufficiently large seed field to explain the present values of the galactic field through a dynamo mechanism.

In this situation, the obvious question is about the effective Lagrangian in the opposite regime $\mathcal{R} \gg m^{2}$. We address this issue in Section 3. Using an improved version of the SDWE [11], we will show that, in the leading-logarithm approximation, the effective coupling is of

the form $F_{\mu \nu} F^{\mu \nu} \ln \frac{R}{\mu^{2}}$. We will also compute the amplitude of the magnetic field produced by this coupling. Unfortunately, since the logarithm is a slowly varying function, this amplitude will be extremely small.

In Section 4 we discuss the case of QED with conformally-invariant quantum fields [6]. Again, we will find that the value of $r$ is too small to be the seed of the galactic dynamo. Throughout the paper we will use units in which $\hbar=c=1$.

\section{PRIMORDIAL FIELDS AND THE SCHWINGER DEWITT EXPANSION}

The Lagrangian for scalar QED on curved backgrounds is given by

\footnotetext{
${ }^{1}$ We denote by $\mathcal{R}$ any component of the Riemann tensor
} 


$$
\mathcal{L}=-\frac{1}{4} F_{\mu \nu} F^{\mu \nu}-D_{\mu} \phi D^{\mu} \phi^{*}+m^{2} \phi \phi^{*}+\xi R \phi \phi^{*}
$$

where $D_{\mu}=\partial_{\mu}-i e A_{\mu}$ is the covariant derivative for the scalar field. The theory is conformally invariant only for massless and conformally coupled $\left(\xi=\frac{1}{6}\right)$ fields.

One can compute an effective Lagrangian for the electromagnetic field by integrating out the quantum scalar field. Using dimensional regularization, the effective Lagrangian can be expanded as follows [9]

$$
\mathcal{L}_{e f f}=-\frac{1}{4} F_{\mu \nu} F^{\mu \nu}+\frac{1}{2} \frac{1}{(4 \pi)^{\frac{d}{2}}}\left(\frac{m}{\mu}\right)^{d-4} \sum_{j=0}^{\infty} a_{j}(x) m^{4-2 j} \Gamma\left(j-\frac{d}{2}\right)
$$

where the $a_{j}(x)$ are the SDW coefficients, and $d$ is the spacetime-dimension. The first three terms are divergent in the limit $d \rightarrow 4$, and the poles must be absorbed into the bare constants of the classical Lagrangian and into a redefinition of the electromagnetic field.

The first SDW coefficients for a charged scalar field are given by [10]

$$
\begin{aligned}
a_{0}= & 1 \\
a_{1}= & -\left(\xi-\frac{1}{6}\right) R \\
a_{2}= & \frac{1}{180}\left(R_{\mu \nu \alpha \beta} R^{\mu \nu \alpha \beta}-R_{\mu \nu} R^{\mu \nu}\right)+ \\
& +\frac{1}{2}\left(\xi-\frac{1}{6}\right)^{2} R^{2}+\frac{1}{6}\left(\xi-\frac{1}{5}\right) \square R-\frac{e^{2}}{12} F_{\mu \nu} F^{\mu \nu} \\
a_{3}= & -\frac{e^{2}}{45} F_{\mu \nu ; \rho} F^{\mu \nu ; \rho}-\frac{e^{2}}{180} F_{; \nu}^{\mu \nu} F_{\mu \rho}^{; \rho} \\
& -\frac{e^{2}}{30}\left(\square F_{\mu \nu}\right) F^{\mu \nu}+\frac{e^{3}}{30} F_{\mu \nu} F^{\nu \rho} F_{\rho}^{\mu} \\
& +\frac{e^{2}}{60} R_{\mu \nu \rho \sigma} F^{\mu \nu} F^{\rho \sigma}-\frac{e^{2}}{90} R_{\mu \nu} F^{\mu \rho} F_{\rho}^{\nu} \\
& +\frac{e^{2}}{72} R F_{\mu \nu} F^{\mu \nu}-\frac{e^{2}}{12} \xi R F_{\mu \nu} F^{\mu \nu}+\cdots
\end{aligned}
$$

where we omitted the purely gravitational terms in $a_{3}$.

After absorbing the poles, the effective Lagrangian contains the usual Maxwell term plus the finite corrections

$$
a_{0} m^{4} \ln \frac{m^{2}}{\mu^{2}}+a_{1} m^{2} \ln \frac{m^{2}}{\mu^{2}}+a_{2} \ln \frac{m^{2}}{\mu^{2}}+\sum_{k \geq 3} \frac{a_{k}}{\left(m^{2}\right)^{k-2}} \Gamma(k-2)
$$


From the above equations we see that the first non trivial interactions between the gravitational and electromagnetic fields are contained in $a_{3}$ and read

$$
R F_{\mu \nu} F^{\mu \nu}, R_{\mu \nu \rho \sigma} F^{\mu \nu} F^{\rho \sigma}, R_{\mu \nu} F^{\mu \rho} F_{\rho}^{\nu}
$$

In the same fashion, the SDW coefficient $a_{n+2}$ contains interactions that can be (schematically) written as $\frac{(\nabla)^{p} \mathcal{R}^{n-p}}{m^{2 n}} F^{2}$.

In what follows, we will analyze the magnetic field produced by a typical term in the SDWE. For simplicity we consider the following effective Lagrangian

$$
\mathcal{L}_{e f f}=-\frac{1}{4} F_{\mu \nu} F^{\mu \nu}\left(1+b\left(\frac{R}{m^{2}}\right)^{n}\right)
$$

The classical equations of motion are given by

$$
\nabla^{\mu}\left(F_{\mu \nu}\left(1+b\left(\frac{R}{m^{2}}\right)^{n}\right)=0\right.
$$

In the particular case of a spatially flat Robertson Walker metric

$$
d s^{2}=a^{2}(\eta)\left(-d \eta^{2}+d x^{2}+d y^{2}+d z^{2}\right)
$$

they read

$$
\partial^{\mu}\left(F_{\mu \nu}\left(1+b\left(\frac{R}{m^{2}}\right)^{n}\right)=0\right.
$$

Obviously, if $R$ is constant (exponential inflation), these equations do not differ from Maxwell equations. Therefore, non trivial effects appear only for extended inflation.

We will work in the radiation gauge $A_{0}=\sum_{i=1}^{3} \partial_{i} A_{i}=0$ The Fourier expansion of the field is

$$
A_{j}(\eta, \vec{x})=\int \frac{d^{3} k}{(2 \pi)^{3} 2 \omega} \sum_{\lambda=1}^{2} \varepsilon_{j}^{(\lambda)}(\vec{k})\left[a^{(\lambda)}(\vec{k}) A_{k}(\eta) e^{i \vec{k} . \vec{x}}+\text { h.c. }\right]
$$

where $\omega=k, a^{(\lambda)}(\vec{k})$ and $a^{\dagger(\lambda)}(\vec{k})$ are the usual annihilation and creation operators. The vectors $\vec{\varepsilon}^{(\lambda)}(\vec{k})$ satisfy $\vec{\varepsilon}^{(\lambda)}(\vec{k}) . \vec{k}=0$. The classical equation for the Fourier modes reads

$$
\left(\ddot{A}_{k}+k^{2} A_{k}\right)\left(1+b\left(\frac{R}{m^{2}}\right)^{n}\right)+\frac{b n}{\left(m^{2}\right)^{n}} R^{n-1} \dot{A}_{k} \dot{R}=0
$$


where the dots denote derivatives with respect to the conformal time $\eta$.

During the inflationary period, $R \gg m^{2}$ and the quantum correction dominates over the Maxwell Lagrangian. Indeed, from Einstein equations we have $R \sim O\left(\rho_{t o t} / m_{p l}^{2}\right)$ where $\rho_{t o t}$ is the total energy density. Consequently, the term $b\left(R / m^{2}\right)^{n} F_{\mu \nu} F^{\mu \nu}$ dominates over the usual $F_{\mu \nu} F^{\mu \nu}$ as long as $\rho_{t o t} \gg m_{p l}^{2} m^{2} b^{-\frac{1}{n}}$. This gives $\rho_{t o t} \gg b^{-\frac{1}{n}}\left(10^{8} \mathrm{GeV}\right)^{4}$ for $m=m_{e}$, the electron mass. In this situation, Eq. 11 reads

$$
\ddot{A}_{k}+k^{2} A_{k}+n \frac{\dot{R}}{R} \dot{A}_{k}=0
$$

Before proceeding, we would like to stress an important point related to the normalization of the Fourier modes $A_{k}$. As our effective Lagrangian is now $-\frac{b}{4}\left(R / m^{2}\right)^{n} F_{\mu \nu} F^{\mu \nu}$, the canonical commutation relations between the coordinates $A_{i}$ and the conjugate momenta $\pi^{i}=\dot{A}^{i} b\left(\frac{R}{m^{2}}\right)^{n} \mathrm{read}$

$$
\left[A_{i}(\eta, \vec{x}), \dot{A}_{j}\left(\eta, \overrightarrow{x^{\prime}}\right) b\left(\frac{R}{m^{2}}\right)^{n}\right]=i\left(\delta_{i j}-\frac{\partial_{i} \partial_{j}}{\nabla^{2}}\right) \delta^{3}\left(\vec{x}-\overrightarrow{x^{\prime}}\right)
$$

The usual commutation relations between the creation and annihilation operators are compatible with the above commutator only if

$$
\dot{A}_{k} A_{k}^{*}-A_{k} \dot{A}_{k}^{*}=\frac{2 \omega i}{b\left(\frac{R}{m^{2}}\right)^{n}}
$$

This will be important in what follows.

Consider the quantity

$$
\rho_{B}=\frac{1}{8 \pi a^{4}}\left\langle B^{2}\right\rangle
$$

where $\langle\cdots\rangle$ denotes the vacuum expectation value. Although this is not the magnetic field energy density associated with the effective Lagrangian (6), it becomes the energy density when $\left(\frac{R}{m^{2}}\right)^{n} \ll 1$. As a consequence, we can analyze the time evolution of $\frac{\rho_{B}}{\rho_{t o t}}$ in order to obtain the value of $r$ at a time when the Maxwell Lagrangian dominates. For simplicity, we will refer to $\rho_{B}$ as the magnetic energy density.

In terms of the Fourier modes we have 


$$
\rho_{B}=\frac{1}{8 \pi a^{4}} \int \frac{d^{3} k}{(2 \pi)^{3} 2 \omega} 2 k^{2}\left|A_{k}(\eta)\right|^{2}
$$

The energy density in the $k$ th mode of the magnetic field, defined as $\rho_{B}(k)=k d \rho_{B} / d k$, is given by

$$
\rho_{B}(k)=\frac{1}{16 \pi^{3} a^{4}} k^{4}\left|A_{k}(\eta)\right|^{2}
$$

During extended inflation we can think the Universe as filled with a perfect fluid having an equation of state $p=\gamma \rho$, with $-1<\gamma<-1 / 3$. The scale factor evolves as $a(t)=\left(\frac{t}{T}\right)^{\alpha}$ where $\alpha=2 / 3(1+\gamma)>1$ and $T$ is chosen so that $a_{\text {today }}=1$. This implies that, in conformal time, $\dot{R} / R=2 /(\alpha-1) \eta$. For this evolution, the properly normalized solution of Eq.12 is given by

$$
A_{k}(\eta)=(-k \eta)^{\nu} \sqrt{\frac{\pi}{b}} e^{i\left(\nu+\frac{1}{2}\right) \frac{\pi}{2}}\left(\frac{m^{2} T}{6 \alpha(2 \alpha-1)}\right)^{\frac{n}{2}}\left(\frac{k T}{\alpha-1}\right)^{\frac{n}{\alpha-1}} H_{\nu}^{(1)}(-k \eta)
$$

where $\nu=\frac{1}{2}-\frac{n}{\alpha-1}$ and $H_{\nu}^{(1)}(x)$ is a Hankel function. In order to select a unique solution we have also imposed that $A_{k}(\eta) \sim e^{-i k \eta}$ as $\eta \rightarrow-\infty$. This is the natural choice for the vacuum state in the in region.

First horizon crossing takes place when $-k \eta=2 \pi \alpha /(\alpha-1)$. Inserting this value of $\eta$ in Eqns.17 and 18 one finds the value of $\rho_{B}$ at that time. The value of $\rho_{\text {tot }}(\eta)$ can be obtained from Einstein equations at first horizon crossing. It is given by

$$
\rho_{t o t}(\lambda)=\frac{m_{p l}^{2}}{6 \pi}\left(\frac{4 \pi}{3}\right)^{\frac{2}{\alpha-1}}\left(\frac{3 \alpha}{2}\right)^{\frac{2 \alpha}{\alpha-1}}\left(\frac{\lambda}{2 \pi T}\right)^{\frac{2}{\alpha-1}} T^{-2}
$$

The value of $r$ at first horizon crossing, denoted by $r_{0}$, is therefore given by $r_{0}=\left.\frac{\rho_{B}}{\rho_{\text {tot }}}\right|_{1^{\text {st }} \text { crossing }}$. After first horizon crossing the quantum fluctuations are assumed to become classical perturbations. For $k \eta \gg 1$ the term $k^{2} A_{k}$ in Eq.12 can be neglected. The time evolution of the Fourier modes is approximately given by

$$
\ddot{A}_{k}+\frac{n s}{\eta} \dot{A}_{k}=0
$$

where $s=-6(1+\gamma) /(1+3 \gamma)$. 
From here, the analysis follows closely that of Ref. [4. Eq.20 admits a constant solution, which gives an uninteresting $\rho_{B} \sim a^{-4}$. It also has the solution

$$
A_{k} \sim \eta^{\frac{[(6 n+1)+(6 n+3) \gamma]}{1+3 \gamma}} \sim(a)^{\frac{1}{2}[(6 n+1)+(6 n+3) \gamma]}
$$

The solution grows with time for $\gamma>-\frac{6 n+1}{6 n+3}$. In this situation, and as long as $R \gg m^{2}$, $r$ evolves like $\rho_{t o t}^{-\frac{2 n+2 \gamma(n+1)}{1+\gamma}}$. When $R \simeq m^{2}$, or when conductivity effects during reheating [4] become dominant, $\rho_{B} \sim a^{-4}$ and $r \propto \rho_{\text {tot }}^{\frac{(1-3 \gamma)}{3(1+\gamma)}}$. Assuming a very rapid reheating, the Universe enters the radiation dominated period, $\rho_{t o t}$ equals $\rho_{\gamma}$, and $r$ becomes a constant. In order to calculate this final value of $r$ we have to distinguish between two possibilities. The first one corresponds to the case in which the growing solution disappears before the plasma effects become dominant. This means that $M^{4}<b^{-\frac{1}{n}} m_{p l}^{2} m^{2}$, where $M^{4}$ is the total energy density at the end of the inflationary period, and $b^{-\frac{1}{n}} m_{p l}^{2} m^{2}$ is the total energy density at the instant in which the $R^{n} F^{2}$ term ceases to dominate over the usual Maxwell term. In this case, and using the relations betwen $r$ and $\rho_{\text {tot }}$ stated above, we get that the value of $r$ at the beginning of the radiation dominated period is given by

$$
r=r_{0}\left(\frac{\rho_{t o t}(k)}{b^{-\frac{1}{n}} m_{p l}^{2} m^{2}}\right)^{\frac{2 n+2(n+1) \gamma}{1+\gamma}}\left(\frac{M^{4}}{b^{-\frac{1}{n}} m_{p l}^{2} m^{2}}\right)^{\frac{1-3 \gamma}{3(1+\gamma)}}
$$

The other possibility is that the conductivity becomes important before the growing solution disappears, i.e., that $M^{4}>b^{-\frac{1}{n}} m_{p l}^{2} m^{2}$. Then, $r \propto \rho_{t o t}^{-\frac{2 n+2 \gamma(n+1)}{1+\gamma}}$ during all the period that goes from first horizon crossing to the end of inflation, and therefore the final value of $r$ reads now

$$
r=r_{0}\left(\frac{\rho_{t o t}(k)}{M^{4}}\right)^{\frac{2 n+2(n+1) \gamma}{1+\gamma}}
$$

For $b=O(1)$ and $m=m_{e}$, we found that there is a value of $\gamma$ between -0.7 and -0.6 that separates the two cases. The results for different values of $\gamma, \lambda=1 M p c$ and $m=m_{e}$ are summarized in Table I. 


\begin{tabular}{|c|c|c|c|c|c|}
\hline \multicolumn{5}{|c|}{ Table I } \\
\hline$\gamma$ & \multicolumn{4}{|c|}{$\left.r\right|_{1 M p c}$} \\
\hline & $n=1$ & $n=2$ & $n=3$ & $n=4$ & $n=5$ \\
\hline-0.5 & $10^{-84}$ & $10^{-67}$ & $10^{-51}$ & $10^{-35}$ & $10^{-19}$ \\
-0.6 & $10^{-92}$ & $10^{-63}$ & $10^{-37}$ & $10^{-10}$ & $10^{17}$ \\
-0.7 & $10^{-116}$ & $10^{-96}$ & $10^{-77}$ & $10^{-56}$ & $10^{-36}$ \\
-0.8 & $\left(10^{-141}\right)$ & $10^{-146}$ & $10^{-147}$ & $10^{-148}$ & $10^{-149}$ \\
-0.9 & $\left(10^{-184}\right)$ & $\left(10^{-222}\right)$ & $10^{-253}$ & $10^{-269}$ & $10^{-285}$ \\
\hline
\end{tabular}

Table I: $\left.r\right|_{1 M p c}$ as a function of $\gamma$ and $n$. The numbers between brackets correspond to situations where there is no growing solution. The value of $r$ for $n=5$ and $\gamma=-0.6$ cannot be trusted because it does not satisfy the condition $r \ll 1$.

These results depends strongly on $n$. For $\gamma>-.6$, we are in the case in which the growing solution disappears before the conductivity becomes dominant. We can see from Table I and from Eq.22 that the value of $r$ increases exponentially with $n$ (for $n$ high enough, the resultant field can serve to seed the galactic dynamo). For $\gamma<-.7$, plasma effects dominates before the growing solution disappears, and therefore the period of time during which the amplification mechanism is operative, is less than the corresponding one in the opposite case. Then, the value of $r$ as given by Eq23, is a compromise between the amplification and the time during which it takes place. In Table I we can see this behavior: for $\gamma=-.7$ we still have an exponential growth of $r$, while for $\gamma<-.8$ the period of amplification is not enough and $r$ now decreases exponentially with $n$.

It may seem strange that we obtain an exponential decrease in certain cases while we can see from Eqs.22 and 23 that the amplification factor grows exponentially with $n$. The cause of this behavior is in the dependence of the initial condition $r_{0}$ on $n$ (see Table II). 


\begin{tabular}{|c|c|c|c|c|c|}
\hline \multicolumn{5}{|c|}{ Table II } \\
\hline$\gamma$ & \multicolumn{5}{|c|}{$\left.r_{0}\right|_{1 ~ M p c}$} \\
\hline & $n=1$ & $n=2$ & $n=3$ & $n=4$ & $n=5$ \\
\hline-0.5 & $10^{-41}$ & $10^{-58}$ & $10^{-76}$ & $10^{-94}$ & $10^{-112}$ \\
-0.6 & $10^{-43}$ & $10^{-71}$ & $10^{-100}$ & $10^{-128}$ & $10^{-157}$ \\
-0.7 & $10^{-44}$ & $10^{-78}$ & $10^{-112}$ & $10^{-146}$ & $10^{-180}$ \\
-0.8 & $10^{-43}$ & $10^{-78}$ & $10^{-113}$ & $10^{-148}$ & $10^{-183}$ \\
-0.9 & $10^{-45}$ & $10^{-83}$ & $10^{-121}$ & $10^{-159}$ & $10^{-197}$ \\
\hline
\end{tabular}

Table II : $\left.r_{0}\right|_{1 M p c}$ for the electromagnetic field, for different values of $\gamma$ and $n$

As we see, $r_{0}$ decreases exponentially with $n$, so the behavior of the final value of $r$ with $n$ is a compromise between the decrease of the initial condition and the growth of the amplification factor, both of which depend on $\gamma$.

At this point, we would like to compare our results for $n=1$, with those of Ref. [4]. Our values for $r$ are smaller by several orders of magnitude (compare our $r(\gamma=-.6) \sim 10^{-92}$ with the correspondent result $r \sim 10^{-68}$ in Ref. [4]). It is easy to find the origin of the discrepancy. The calculation in Ref. [4] begins after first horizon crossing and assumes that the initial value of $\rho_{B}$ is $H^{4}$. This is the value of the energy density for a massless minimally coupled scalar field. Using this value the resulting $r_{0}$ varies between $10^{-33}$ and $10^{-12}$ (for $-0.9 \leq \gamma<-0.5)$. Here we computed $\left.\rho_{B}\right|_{1 \text { stcrossing }}$ from first principles, and found much smaller values (see Table II). The physical origin of the discrepancy is the normalization condition Eq.14.

Up to here, we have made our calculations using $m=m_{e}$. Now we turn to study the dependence on $m$ of our results. The mass $m$ enters the calculation at two different points. First, it determines when the $\left(\frac{R}{m^{2}}\right)^{n} F^{2}$ coupling ceases to dominate over the usual Maxwell term, and hence it contributes to determine the amount of amplification that will take place. Second, it appears in the initial condition in such a way that $r_{0} \propto m^{2 n}$. We can see that 
raising the value of $m$ improves the initial condition $r_{0}$. On the other hand, it makes shorter the amplification period, and the final result is a compromise between these two effects. We have analysed the mass dependence taking these facts into account. We have found that

for $M^{2}<b^{-\frac{1}{n}} m_{p l} m$, the results decrease with increasing mass, while for $M^{2}>b^{-\frac{1}{n}} m_{p l} m$ the results increase with mass. In the first case the shortening of the amplification period preponderates over the increasing of the initial condition, making the result decreasing with increasing mass. In the other case, the duration of the amplification period depends only on the value of $M$ and no longer on $m$. This means that raising the value of the mass improves the initial condition but does not affect the amplification, giving an increasing result with increasing mass. However, in this case the value of $m$ cannot be raised arbitrarily, because it must obey the relation $M^{2}>b^{-\frac{1}{n}} m_{p l} m$.

To summarize, although the value of $r_{0}$ is strongly suppressed with respect to that of a minimally coupled scalar field, and although the suppression increases with $n$, there are values of $\gamma$ such that, after first horizon crossing, a large amplification takes place, and produces final values of $r$ large enough to serve as the seed magnetic field.

\section{IMPROVING THE SDWE - KILLING THE MAGNETIC FIELD}

From a phenomenological point of view, the couplings discussed in the previous section may help to solve the problem of the generation of a primordial field whitout breaking gauge invariance. Do they have a theoretical motivation? In order to answer this question, we need to compute the effective action for the electromagnetic field in the limit $\mathcal{R} \gg m^{2}$. To do this, we will use the improved version of the SDWE developed by Parker and Toms in Ref. [1].

A partial ressumation of the SDWE can be achieved by doing the expansion in inverse powers of $\tilde{m}^{2}=m^{2}+\left(\xi-\frac{1}{6}\right) R$ instead of inverse powers of $m^{2}$. In this case, as conjectured in Ref. [11] and proved in Ref. [12], the new SDW coefficients (denoted here by $b_{j}$ ) do not contain powers of the scalar curvature $R$. The first $b_{j}$ are given by 


$$
\begin{aligned}
b_{0}= & 1 \\
b_{1}= & 0 \\
b_{2}= & \frac{1}{180}\left(R_{\mu \nu \alpha \beta} R^{\mu \nu \alpha \beta}-R_{\mu \nu} R^{\mu \nu}\right)+\frac{1}{6}\left(\xi-\frac{1}{5}\right) \square R-\frac{e^{2}}{12} F_{\mu \nu} F^{\mu \nu} \\
b_{3}= & -\frac{e^{2}}{45} F_{\mu \nu ; \rho} F^{\mu \nu ; \rho}-\frac{e^{2}}{180} F_{; \nu}^{\mu \nu} F_{\mu \rho}^{; \rho}- \\
& -\frac{e^{2}}{30}\left(\square F_{\mu \nu}\right) F^{\mu \nu}+\frac{e^{3}}{30} F_{\mu \nu} F^{\nu \rho} F_{\rho}^{\mu}+ \\
& +\frac{e^{2}}{60} R_{\mu \nu \rho \sigma} F^{\mu \nu} F^{\rho \sigma}-\frac{e^{2}}{90} R_{\mu \nu} F^{\mu \rho} F_{\rho}^{\nu}+\cdots
\end{aligned}
$$

where we did not include the purely gravitational terms in $b_{3}$. Note that $b_{1}$ and $b_{2}$ do not contain the terms proportional to $R$ and $R^{2}$ that are present in $a_{1}$ and $a_{2}$ (see Eq.3). The same thing happens for all $b_{j}$.

The improved expansion can be obtained from the usual SDWE (Eq.2) substituting $m^{2}$ by $\tilde{m}^{2}$ and the coefficients $a_{j}$ by the $b_{j}$. In the limit $\left(\xi-\frac{1}{6}\right) R \gg m^{2}$, the coefficient $b_{2}$ induces a coupling of the form

$$
\frac{1}{4}\left(\frac{e^{2}}{96 \pi^{2}}\right) F_{\mu \nu} F^{\mu \nu} \ln \left(\frac{R}{\mu^{2}}\right)
$$

Under the assumption $\ln \frac{R}{\mu^{2}} \gg 1$, this term dominates and the effective lagrangian reduces to

$$
\mathcal{L}_{e f f}=-\frac{1}{4} F_{\mu \nu} F^{\mu \nu}\left(1-\frac{e^{2}}{96 \pi^{2}} \ln \frac{R}{\mu^{2}}\right)+\ldots
$$

where $\mu$ is an arbitrary scale. It is worth noting that this result is also valid beyond one-loop, in the leading-logarithm approximation. A similar effective lagrangian has been proposed for non-abelian gauge theories in Ref. [13].

There is a simple physical interpretation of the effective Lagrangian given by Eq. 26. If we couple $\mathcal{L}_{\text {eff }}$ to an external current, $\mathcal{L}_{\text {eff }} \rightarrow \mathcal{L}_{\text {eff }}+e J_{\mu} A^{\mu}$, after a rescaling of $A^{\mu}$ we obtain the usual Maxwell Lagrangian with a running (curvature-dependent) electric charge

$$
e^{2}(R)=\frac{e^{2}\left(\mu^{2}\right)}{1-\frac{e^{2}}{96 \pi^{2}} \ln \frac{R}{\mu^{2}}}
$$

This is the scale dependence dictated by the renormalization group. This interpretation also makes explicit the fact that $\mathcal{L}_{\text {eff }}$ is valid only under the assumption $\frac{e^{2}}{96 \pi^{2}} \ln \frac{R}{\mu^{2}} \ll 1$. 
Otherwise, an analysis of strongly coupled QED is needed (strictly speaking, for such large values of the curvature one should replace QED by a GUT).

We will perform the same calculation we did in the previous Section, that is, calculate the value of $r$ for a scale of $1 M p c$. Typically, this scale crosses outside the horizon not long after curvature falls below the GUT scale $\left(10^{28} \mathrm{GeV}^{2}\right)$. Thus, we cannot use the effective lagrangian (26) to calculate the initial condition, so we will consider $r_{0}$ as given and study its evolution after first horizon crossing.

The equation for Fourier modes is

$$
\ddot{A}_{k}+k^{2} A_{k}-\frac{e^{2}}{96 \pi^{2}} \frac{\dot{R}}{R} \frac{\dot{A}_{k}}{\left(1-\frac{e^{2}}{96 \pi^{2}} \ln \frac{R}{\mu^{2}}\right)}=0
$$

We will take $\mu^{2}=R_{R H}$, the value of the scalar curvature at the end of reheating, and we will consider that $e$, the electric charge when $R=R_{R H}$, is of the same order of magnitude of its value today. Then we can assure that, during most of inflation, $\ln \frac{R}{R_{R H}} \gg 1$ and $\frac{e^{2}}{96 \pi^{2}} \ln \frac{R}{R_{R H}} \ll 1$, the conditions needed for the validity of the leading logarithm approximation. In this situation, for modes outside the horizon, we can approximate Eq. 28 by

$$
\ddot{A}_{k}-\frac{e^{2}}{96 \pi^{2}} \frac{\dot{R}}{R} \dot{A}_{k}=0
$$

This equation has the same form of Eq. 20, if we make the identification $n \leftrightarrow-\frac{e^{2}}{96 \pi^{2}} \equiv x$. The solutions are the same as those of Eq. 20, that is, $A_{k}=$ constant, which gives $\rho_{B} \propto a^{-4}$, and

$$
A_{k} \sim(a)^{\frac{1}{2}[(6 x+1)+(6 x+3) \gamma]}
$$

Using that $x \sim 10^{-4}$ we can write Eq. 30 as

$$
A_{k} \sim(a)^{\frac{1}{2}[1+3 \gamma]}
$$

Since $1+3 \gamma<0$, this means that, for this solution, $\rho_{B}$ decreases more rapidly than $a^{-4}$. So we can consider only the contribution of the constant solution and conclude that for the effective lagrangian given by Eq. 26, $\rho_{B}$ decreases as $a^{-4}$, and then $r \propto \rho_{\text {tot }}^{\frac{(1-3 \gamma)}{3(1+\gamma)}}$. 
The final value of $r$ will be

$$
r=r_{0}\left(\frac{M^{4}}{\rho_{t o t}(k)}\right)^{\frac{(1-3 \gamma)}{3(1+\gamma)}}
$$

For $\gamma<-0.5$, the amplification factor that accompanies $r_{0}$ is smaller than $10^{-49}$. Since the initial condition is tipically much less than 1 , the value of $r$ is in all cases very far from the required value $10^{-34}$.

To conclude, although the higher order terms in the SDWE $\mathcal{R}^{n} F^{2}$ generate large amplitudes for the magnetic field, once these terms are ressumed they generate only a logarithmic interaction $\ln R F^{2}$. This interaction produces a discouragingly small seed field.

\section{TRACE ANOMALY AND PRIMORDIAL FIELDS}

We end the paper with a comment about the conformally invariant case $m^{2}=0, \xi=1 / 6$. Of course in this situation neither the SDWE nor its improved version are useful to compute the effective Lagrangian. However, it is easy to find a closed expression for it.

It is well known that conformal invariance is broken by quantum effects. The (anomalous) trace of the energy momentum tensor is given by the second SDW coefficient [9]

$$
T_{\mu}^{\mu}=-\frac{a_{2}}{16 \pi^{2}}=\frac{e^{2}}{192 \pi^{2}} F_{\mu \nu} F^{\mu \nu}+\ldots
$$

where the dots denote purely gravitational terms proportional to $\mathcal{R}^{2}$ and $\nabla \nabla \mathcal{R}$.

In a Robertson Walker space-time, the above trace anomaly can be derived from the effective Lagrangian [14]

$$
\mathcal{L}_{e f f}=-\frac{1}{4} F_{\mu \nu} F^{\mu \nu}\left(1+\kappa \ln \frac{a}{a_{0}}\right)+\ldots
$$

where $\kappa=\frac{e^{2}}{48 \pi^{2}}$ and $a_{0}$ is a reference value for the scale factor (for example the value of $a$ after inflation). Here the dots denote non-local terms independent of the scale factor. 1

\footnotetext{
${ }^{2}$ There is a sign difference between the logarithmic term of the effective lagrangian in Ref. [14] and that of Ref. [6]. We agree with the result of Ref. [14].
} 
As before, Eq. 34 can be interpreted as the usual Maxwell Lagrangian with a scale dependent electric charge

$$
e^{2}(a)=\frac{e^{2}\left(a_{0}\right)}{\left(1+\kappa \ln \frac{a}{a_{0}}\right)}
$$

The effective Lagrangian is valid as long as $\kappa \ln \frac{a}{a_{0}} \ll 1$.

Under this assumption, one can estimate the magnetic field generated by the conformal anomaly. The resulting value for $r$ is again extremely small, of order $10^{-104}$ for $\lambda \sim 1 M p c$.

It has been argued [6] that this could be an efficient mechanism if a large number of massless $(m \ll H)$ fields are present during inflation, so that $\kappa \rightarrow N \kappa \sim O(1)$. Using the approximation

$$
\mathcal{L}_{e f f} \simeq-\frac{1}{4} F_{\mu \nu} F^{\mu \nu}\left(\frac{a}{a_{0}}\right)^{N \kappa}
$$

it is possible to show [6] that the previous result for $r$ is multiplied by a huge amplification factor $A=\left(\frac{H}{k}\right)^{N \kappa} \sim 10^{50 N \kappa}$ for $H=10^{12} \mathrm{GeV}$ and $\lambda=1 \mathrm{Mpc}$.

However, during that period $\ln \frac{a}{a_{0}}$ varies between -60 and 0 and the approximation (36) breaks down for $N \kappa \sim 1$. In this situation, even the effective Lagrangian (34) is inadequate,

since at some point $1+N \kappa \ln \frac{a}{a_{0}}$ vanishes and one reaches the Landau singularity. Therefore, the conclusion is that, if $N \kappa$ is sufficiently small in order to assure that $N \kappa \ln \frac{a}{a_{0}} \ll 1$ during the period of interest, the amplification factor is very small, $A \simeq 1+N \kappa \ln \frac{H}{k}$. The amplitude of the magnetic field generated is too small to be of astrophysical interest. On the other hand, if $N \kappa \sim O(1)$, the final result for $r$ would depend on the physics of QED at strong coupling.

\section{ACKNOWLEDGMENTS}

We would like to thank D.D. Harari, M. Zaldarriaga and E. Calzetta for useful conversations. This research was supported by Universidad de Buenos Aires, Consejo Nacional de Investigaciones Científicas y Técnicas and by Fundación Antorchas. 


\section{REFERENCES}

[1] E.N. Parker, Cosmical Magnetic Fields, Clarendon Press, (1979).

[2] Ya.B. Zel'dovich, A.A. Ruzmaikin, and D.D. Sokoloff, Magnetic Fields in Astrophysics, Gordon and Breach, New York, (1983).

[3] E.R. Harrison, Phys. Rev. D30,188,(1973).

[4] M.S. Turner and L.M. Widrow, Phys. Rev. D37,2743,(1987).

[5] B. Ratra, Caltech preprint GPR-287/CALT-68-1751,(1991); B. Ratra, Astrophys. Jour. 391,L1,(1992); M.Gasperini, M.Giovannini and G.Veneziano, CERN-TH/95-85 (1995)

[6] A.D. Dolgov, Phys. Rev. D48,2499, (1993).

[7] I.T. Drummond and S.J. Hathrell, Phys. Rev. D22,343, (1980).

[8] B.S. De Witt, Dynamical Theory of Groups and Fields, Gordon and Breach, New York, (1965).

[9] N.D. Birrell and P.C.W Davies, Quantum Fields in Curved Space, Cambridge University Press, (1982).

[10] A.O. Barvinsky and G.A. Vilkovisky, Phys. Rep. 119C,1 (1985)

[11] L. Parker and D.J Toms, Phys. Rev. D31,953,(1985).

[12] I. Jack and L. Parker, Phys. Rev. D31,2439,(1985).

[13] E. Calzetta, I. Jack and L. Parker, Phys. Rev. D33,953,(1986).

[14] A.D. Dolgov, Sov. Phys. JETP54,223, (1981). 